\title{
Interação da Gestação na Atividade da Doença Inflamatória Intestinal e sua Influência sobre o Prognóstico Gestacional e na Fecundidade
}

\author{
Interaction of Pregnancy on Inflammatory Bowel Disease Activity and its \\ Influence on Pregnancy Outcome and Fecundity
}

\author{
LEONARDO CORRÊADE OLIVEIRARODRIGUES ${ }^{1}$; MAGALY GEMIOTEIXEIRA-TSBCP²; \\ ROBERTATHIERY GODOYARASHIRO ${ }^{3}$; DESIDÉRIO ROBERTO KISS - TSCBC ${ }^{4}$
}

\begin{abstract}
${ }^{1}$ Pós-graduando do Serviço de Coloproctologia do Departamento de Gastroenterologia do Hospital das Clínicas da Faculdade de Medicina da Universidade de São Paulo - HCFMUSP; ${ }^{2}$ Supervisora do Serviço de Coloproctologia do Departamento de Gastroenterologia do Hospital das Clínicas da Faculdade de Medicina da Universidade de São Paulo - HCFMUSP; ${ }^{3}$ Pós-graduando do Serviço de Coloproctologia do Departamento de Gastroenterologia do Hospital das Clínicas da Faculdade de Medicina da Universidade de São Paulo - HCFMUSP; ${ }^{4}$ Serviço de Coloproctologia do Departamento de Gastroenterologia do Hospital das Clínicas da Faculdade de Medicina da Universidade de São Paulo - HCFMUSP.
\end{abstract}

RODRIGUES LCO; TEIXEIRA MG; ARASHIRO RTG; KISS DR. Interação da Gestação na Atividade da Doença Inflamatória Intestinal e sua Influência sobre o Prognóstico Gestacional e na Fecundidade. Rev bras Coloproct, 2009;29(3): 329-344.

RESUMO: INTRODUÇÃO: A maioria das mulheres que desenvolvem doença inflamatória intestinal (DII) encontra-se em idade fértil, despertando preocupação dos médicos e mulheres no entendimento desta interação. Avaliamos a influência da DII sobre a fecundidade e gestação e vice- versa. MÉTODOS: Os protocolos de pacientes com doença de Crohn (DC) e retocolite ulcerativa (RC), de 1984 a 2006, em idade fértil, cadastrados no ambulatório de DII, foram revisados. Pacientes foram entrevistados para preenchimento de dados não encontrados nos protocolos. Outros tipos de colites, investigação incompleta, pacientes que não estavam em idade fértil ou sem capacidade cognitiva foram excluídos. Prematuridade, baixo peso ao nascer, anomalias congênitas, natimortalidade, abortamentos, tipos de partos, localização da doença na gestante e uso de medicamentos durante a gestação foram investigados. O método estatístico adotado foi o teste de qui- quadrado e Fisher, com nível de significância de $5 \%$. Nenhum paciente se recusou a participar desta pesquisa. RESULTADOS: 140 gestações em 104 pacientes com DII foram avaliados (RC em 63 gestações e DC em 77). Houve redução da fecundidade após o início dos sintomas relacionados à DII em 41,6\%, com influência da doença sobre a opção de não ter filhos em $20,6 \%$ (10,3\% dos pacientes por medo da doença; 6,5\% por orientação médica e $2,2 \%$ por más condições clínicas), sem diferenças entre DC e RC. A grande maioria não quis engravidar por já ter filhos, por ser solteira ou estar ter baixa idade $(53,3 \%)$. A atividade da $\mathrm{RC}$ não foi alterada durante a gestação na maioria das pacientes $(\mathbf{7 7 , 8 \%} ; \mathbf{p} \geq \mathbf{0 , 0 0 3})$. A atividade da doença melhorou durante a gestação mais nas gestantes com DC do que nas com RC (p>0.0007). A incidência de prematuros, baixo peso ao nascer e natimortos foi maior quando todo o cólon estava acometido na $\mathrm{RC}$ (p $\leq 0.037$ ). A proporção estimada de prematuros e baixos pesos ao nascer foi de 83,3\% [IC 95\%: 10,3\%; 100,0\%]. Não houve diferença estatística quanto à localização da doença e alterações do feto na $\mathrm{DC}(\mathrm{p} \geq \mathbf{0 , 6 5 1 3})$. Em 21 gestantes foram administrados aminossalicilatos e em 15, corticosteroides. Em 106 gestações, nenhum medicamento foi administrado. Não houve maior taxa de alterações do concepto quando aminossalicilatos ou corticosteroides foram administrados às gestantes com DII $(p \geq 0,17$ and $p \geq 0,1585$, respectivamente). CONCLUSÃO: A DII não influenciou diretamente na fecundidade na grande maioria das pacientes. A gestação influenciou positivamente a evolução da DC, independente do uso de medicamentos. A taxa de prematuridade foi maior nas proles de mães com DC. Houve maior taxa de proles com alterações quando todo o cólon estava comprometido na mãe com RC. A DC influenciou o tipo de parto, apenas nos casos de doença perianal extensa associada à doença colônica.

Descritores: 1. Proctocolite 2. Doença de Crohn 3. Gestação 4. Enteropatias 5. Fertilidade.

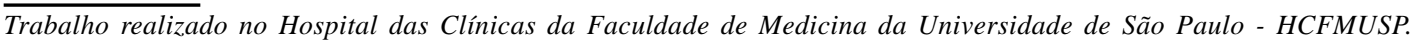




\section{INTRODUÇÃO}

Mais de $50 \%$ das mulheres que desenvolvem a doença inflamatória intestinal (DII) encontra-se em idade fértil. Consequentemente, esta moléstia preocupa pacientes e médicos quanto aos problemas que poderiam ocorrer com a associação à gravidez e a sua influência sobre a fertilidade. Vários estudos têm sido feitos com o intuito de estudar os efeitos da doença inflamatória intestinal sobre a fertilidade e a gestação e vice-versa. Todos estes estudos, no entanto, apresentaram inúmeras limitações metodológicas. Os estudos clássicos iniciais foram realizados nos anos 50, antes da era moderna da terapia medicamentosa. Não havia estratificação dos pacientes, com diferenciação entre a extensão e a gravidade da doença intestinal, suas complicações e tipo de tratamento recebido pelas pacientes.

A gravidez parece ter influência que pode ser ora benéfica ora maléfica, sobre a retocolite ulcerativa (RC) e a doença de Crohn (DC), de acordo com o grau de atividade da doença no momento da concepção $[1,2]$. De um modo geral, os autores têm agrupado os pacientes de acordo com a atividade da doença antes, durante e após a gestação. Outro aspecto que tem sido estudado é o aparecimento da primeira crise da doença inflamatória intestinal durante a gestação. Os resultados têm sido tão discrepantes que De Dombal et al. [3] em trabalho de revisão observaram que nas pacientes com RC em remissão antes da gestação, a taxa de reatividade da doença variou entre $10 \%$ e $54 \%$ durante a gestação. Dois terços das pacientes com inatividade da doença antes da concepção continuam em remissão durante a gravidez. Quando há atividade da doença antes da concepção, estas tendem a permanecer em atividade durante a gestação, com variação entre $25 \%$ e $100 \%$ de piora do quadro clínico, exceto nos casos em que algum tratamento medicamentoso tenha sido iniciado ou que tenha sido aumentada a dose da medicação já em uso [3].

Quanto aos efeitos da RC e da DC sobre a gravidez, estudos demonstraram uma taxa de proles saudáveis, similar à taxa da população em geral $[2,5,6]$. Trabalhos demonstraram que se a doença estiver quiescente durante a gestação, não há aumento do risco de abortamento espontâneo, complicações relacionadas à gestação, anomalias congênitas ou outras intercorrências com o feto [7,8,9,10,11,12,13,14,15]. Estas conclusões não foram confirmadas por outros autores $[16,17]$.
Quando a doença estiver ativa no período da concepção ou durante a gestação, há maior probabilidade do prognóstico ser pior. Nesta situação há maior incidência de abortamento espontâneo, prematuridade e outras alterações perinatais, parecendo ser maior na DC do que na RC. [5,7,13,15,18,19,20,21]. Há trabalhos que demonstraram maior incidência de recémnascidos de baixo peso e pré- termos e risco de abortamento espontâneo em mães com doença inflamatória intestinal, principalmente na DC em atividade, no momento da concepção $[5,18,22,23]$. Alguns trabalhos demonstraram um risco maior de prematuridade antes do diagnóstico nas mães com RC. [24]. As taxas de anomalias congênitas não foram afetadas pela DII. [25].

Nos últimos anos, aumentou significativamente o número de medicamentos utilizados para o tratamento das DII, gerando muitas controvérsias sobre sua utilização durante a gestação, tanto no que concerne aos seus efeitos sobre o concepto quanto aos seus resultados sobre a doença materna. A introdução da terapia biológica no tratamento da doença inflamatória intestinal trouxe novos questionamentos quanto a sua utilização durante a gestação [24,26,27,28].

Aspecto de grande importância refere-se ao estudo dos fatores que influenciam a taxa de natalidade nem sempre relacionados à doença propriamente dita, mas também ao receio dos pais frente a presença de doença grave, crônica e de evolução incerta.

Frente aos inúmeros achados controversos encontrados na literatura, achamos oportuno estudar em nosso meio a influência da doença sobre a fecundidade, ressaltando principalmente os motivos pessoais para as pacientes não engravidarem; se o período da gestação contribuiu para a atividade da DII; se a DII altera a evolução da gestação, incluindo o tipo de parto, se vaginal ou cesáreo, e as influências sobre o concepto (prematuridade, baixo peso, anomalias congênitas e abortamento); a correlação da extensão da DII sobre a evolução da gestação e o concepto; e as alterações ocorridas no feto e na gestação, com o uso de medicamentos utilizados para o tratamento da DII.

\section{OBJETIVO}

O objetivo deste trabalho é avaliar a influência da DII sobre a fecundidade, evolução da gestação e do concepto e o comportamento da DII durante este período. 


\section{ANÁLISE DE DADOS}

Foram incluídos 1550 pacientes no protocolo de atendimento no ambulatório de DII, sendo 815 pacientes do sexo feminino e destas, 104 pacientes tiveram alguma gestação após o início dos sintomas referentes à DII.

As variáveis coletadas neste estudo, por conta do delineamento experimental, são nominais em sua totalidade. Para verificar o nível da associação entre pares de variáveis nominais foi utilizado o teste Quiquadrado de contingência, com o resíduo ajustado para verificar a associação significante entre as categorias das variáveis estudadas e o teste de Fisher. Para avaliar a proporção entre grupos, foi utilizado teste binomial para comparação. O nível de significância adotado foi de $5 \%$ [33].

\section{MÉTODOS}

Foi realizada uma revisão dos protocolos e registros das pacientes atendidas no ambulatório de DII do Serviço do Cólon e Reto do Departamento de Gastroenterologia do Hospital das Clínicas da Faculdade de Medicina da Universidade de São Paulo, no período de 1984 a 2006. A participação das pacientes no estudo obedeceu aos seguintes critérios:

\section{Critérios de inclusão}

- DC ou RC com diagnóstico confirmado.

- Paciente em idade fértil (entre15 e 45 anos).

- Paciente em condições de responder aos quesitos do questionário.

\section{Critérios de exclusão}

indeterminada)

- Outros tipos de colites (p.ex. colite completa.

- Pacientes com investigação diagnóstica in-

- Pacientes fora da idade fértil.

- Pacientes sem capacidades cognitivas.

O período de idade fértil considerado foi da menarca até a menopausa de cada paciente. As entrevistas foram realizadas quando as pacientes retornavam para as consultas agendadas de rotina. No caso de não comparecimento, as pacientes foram convidadas a participar do estudo, através de contato telefônico ou correspondência. $\mathrm{O}$ estudo foi realizado no período de fe- vereiro de 2006 a julho de 2007. Trata- se de um estudo retrospectivo, uma vez que, além das revisões dos prontuários, as entrevistas foram referentes à acontecimentos passados, apenas para complementação de dados não registrados nos protocolos existentes. Nenhuma paciente se recusou a participar do estudo.

Estas revisões e entrevistas visaram obter a história obstétrica, incluindo as intercorrências, as condições clínicas das pacientes e as medicações utilizadas dois meses antes, durante e dois meses após a gestação, o número de abortamentos espontâneos e provocados, e a história do concepto, quanto à presença de anomalias congênitas, prematuridade, peso ao nascimento e natimortalidade. Esses itens foram definidos de acordo com Rezende J. et al [29] :

Prematuridade: quando o parto ocorre antes da $37^{\mathrm{a}}$ semana de gestação.

Baixo peso ao nascer: peso ao nascer entre 500 e 2500 gramas.

Anomalias congênitas: são as anomalias encontradas no concepto, presentes ao nascer, mesmo quando de manifestação tardia, na vida pós- natal.

Natimortalidade: morte natural do feto após a $20^{\mathrm{a}}$ semana de gestação.

Abortamento espontâneo ou natural: óbito fetal intra- útero antes da $20^{\mathrm{a}}$ semana de gestação.

Abortamento provocado: interrupção da gestação, podendo ser por indicação terapêutica, relacionada ou não à RC ou DC.

As análises relacionadas à atividade da DII $\mathrm{e}$ às alterações do concepto, dentre elas a prematuridade, baixo peso ao nascer, anomalias congênitas e natimortalidade, foram realizadas apenas naquelas provenientes de gestantes que já tinham a DII antes da concepção. Quando analisados o abortamento espontâneo ou natural e o provocado, além dos tipos de parto, foram incluídas as gestações de pacientes que apresentaram início dos sintomas da DII durante a gestação.

Foi feito um levantamento dos dados correspondentes à prematuridade, baixo peso ao nascer, anomalias congênitas, natimortalidade, partos vaginais e cesáreos, no estado de São Paulo, no período de 2000 a 2005, na população em geral [30]. Escolhemos esse período pois são os dados mais recentes obtidos no estado de São Paulo, de onde provém a grande maioria das pacientes do estudo.

Cada paciente foi questionada quanto à sua condição clínica nos últimos dois meses que precede- 
ram a concepção, durante cada gestação e durante o puerpério. A condição clínica neste protocolo se resumiu apenas se a doença estava ativa ou inativa, sendo classificadas as pacientes de acordo com os índices de Truelove \& Witts [31] para RC e o índice simplificado de Harvey-Bradshaw [32] para DC. Ambos são índices embasados em critérios clínicos, uma vez que não há registros endoscópicos e anátomo- patológicos das pacientes de todos os períodos analisados neste trabalho, estando a paciente com ou sem a crise.

Durante os três períodos citados, foram registradas as medicações específicas para o tratamento da DII, incluindo os salicilatos, corticosteroides, imunossupressores, antibióticos como o ciprofloxaxino e o metronidazol e infliximabe.

Quanto à investigação da fertilidade, foram investigadas as razões por não engravidar, antes e depois do aparecimento dos sintomas, segundo protocolo em anexo.

Todas as pacientes entrevistadas, que participaram desta pesquisa, assinaram o termo de consentimento livre e esclarecido.

\section{RESULTADOS}

Dos 1550 pacientes cadastrados no ambulatório de DII até a realização da pesquisa, havia 815 mulheres das quais 289 responderam à entrevista no período da pesquisa e enquadravam-se nos critérios de inclusão do nosso estudo. Neste grupo havia 140 pacientes com RC e 149 com DC. Antes do início dos sintomas relacionados à DII, 163 mulheres apresentaram 416 gestações (2,55 gestações/mulher) e após o início dos sintomas, 140 gestações em 104 mulheres. Em 15 gestações, o início dos sintomas da DII ocorreu durante a gestação, totalizando 155 gestações de pacientes com DII (1,49 gestações/mulher).

Das 140 gestações que ocorreram depois do início dos sintomas de DII, 63 gestações ocorreram em pacientes com RC e 77 em DC.

Em 63 gestações de pacientes com RC, a doença estava ativa em 28 e inativa em 35.

Em 77 gestações de pacientes com DC, a doença estava ativa em 20 e inativa em 57.

Das 289 mulheres avaliadas, 58 ficaram grávidas antes dos sintomas, 56 depois do início da DII e 48 apresentaram gestações antes da DII e também depois.

\subsection{Fecundidade}

Observou-se redução de $41,6 \%$ da fecundidade em pacientes com DII quando comparado o número de gestações antes e após o início de sintomas $(2,55$ para 1,49 gestações/mulher). Das 289 mulheres, 104 quiseram engravidar e conseguiram, 5 quiseram engravidar,e não conseguiram $(5 / 109=4,6 \%)$ e 180 não quiseram engravidar. Os motivos que levaram as pacientes a não desejar a gravidez figuram na tabela 1 :

Tabela 1 - Distribuição das doentes com DII e as razões que as levaram a optar por não engravidar.

\begin{tabular}{|c|c|c|c|c|c|c|c|}
\hline & \multirow[t]{2}{*}{$\begin{array}{l}\text { Motivos para } \\
\text { não engravidar }\end{array}$} & \multicolumn{2}{|c|}{$\begin{array}{l}\text { Retocolite } \\
\text { Ulcerativa }\end{array}$} & \multicolumn{2}{|c|}{$\begin{array}{l}\text { Doença } \\
\text { de Crohn }\end{array}$} & \multicolumn{2}{|c|}{ Total } \\
\hline & & $\mathbf{n}$ & $\%$ & $\mathbf{n}$ & $\%$ & $\mathbf{n}$ & $\%$ \\
\hline \multirow{6}{*}{ Independente da ação do paciente } & Solteira / Idade precoce & 31 & 34,4 & 13 & 14,4 & 44 & 24,4 \\
\hline & Idade Avançada & 6 & 6,7 & 12 & 13,3 & 18 & 10,0 \\
\hline & Orientação médica & 6 & 6,7 & 6 & 6,7 & 12 & 6,7 \\
\hline & Más condições clínicas & 4 & 4,4 & 3 & 3,3 & 7 & 3,9 \\
\hline & Separada & - & - & 7 & 7,8 & 7 & 3,9 \\
\hline & Problemas psiquiátricos & - & - & 2 & 2,2 & 2 & 1,1 \\
\hline \multirow{5}{*}{ Opção da paciente } & Já tinha filhos & 22 & 24,4 & 30 & 33,3 & 52 & 28,9 \\
\hline & Medo da doença & 9 & 10,0 & 10 & 11,1 & 19 & 10,6 \\
\hline & Não quis ter nenhum filho & 5 & 5,5 & 3 & 3,3 & 8 & 4,4 \\
\hline & Trabalha muito & 4 & 4,4 & 3 & 3,3 & 7 & 3,9 \\
\hline & Problemas conjugais & 3 & 3,3 & 1 & 1,1 & 4 & 2,2 \\
\hline Total & & 90 & 100,0 & 90 & 100,0 & 180 & 100,0 \\
\hline
\end{tabular}


Dentre as pacientes que não engravidaram, $21,1 \%$ (38 em 180 pacientes) foram influenciadas por fatores ligados à doença, ou seja, a orientação médica, as más condições clínicas e o medo da doença. Não houve diferença entre as duas doenças $(\mathrm{RC}=21,1 \% \mathrm{e}$ $\mathrm{DC}=21,1 \%$ ).

Houve diferença significativa entre os motivos para não engravidar e o tipo de doença. Nas pacientes com RC, o motivo relatado mais frequente foi o fato das doentes serem muito jovens ou solteiras $\left(x^{2}=21,629\right.$ $\mathrm{p}=0,017)$. Não houve diferença significativa entre os tipos de doença, nos casos em que a escolha entre ter filhos ou não foi opção da paciente.

Quando comparamos os grupos que foram influenciados pela doença e os que não foram, houve diferença estatística, tanto na RC quanto na DC ( $p<0,0001$ ), sendo maior o grupo que não houve influência da doença como motivo para não engravidar.

\subsection{Efeitos da gestação sobre a DII}

De uma maneira geral, as doentes com DII em sua maioria permaneceram inalteradas quanto à atividade inflamatória durante a gestação (vide tabela 2). Verificou-se que na DC, a porcentagem de doentes que melhoraram da sintomatologia durante a gravidez foi significativamente maior do que nos casos de doentes com $\mathrm{RC}\left(\mathrm{x}^{2}=13.255, \mathrm{p}=0,001\right)$. Entretanto não houve diferença significativa quanto aos grupos que tiveram piora ou ficaram inalterados durante gestação $\left(\mathrm{x}^{2}=\right.$ 1,231, $\mathrm{p}=0,59)$. Os dados referentes à atividade da DC e da RCU durante a gestação figuram nas tabelas 2 e tabela 3 .

Houve diferença significativa entre a atividade e o tipo de doença $\left(x^{2}=9,998, p=0,003\right)$. A análise dos resíduos mostra que esta diferença ocorre somente na RC e não na DC. Foram encontradas diferenças significantes entre o tipo de doença e a atividade da DII, quando se verifica a presença ou ausência de alterações no quadro clínico $\left(x^{2}=11,387, p=0,001\right)$.

Houve diferença significativa na probabilidade de melhora entre DC e RC ( $p=0,0007)$, sendo maior na DC. A análise dos resíduos ajustados mostrou que a maioria das pacientes com DC $(74,0 \%)$ apresentou melhora ou permaneceram inalteradas sem atividade da doença, antes e após a concepção. As pacientes com RC apresentaram-se, em sua grande maioria, inalteradas $(77,8 \%)$, independente da atividade da doença, antes da concepção. Não houve diferença significativa na chance de piora entre DC e RC $(\mathrm{p}=0,9999)$.

Tabela 2 - Distribuição das doentes, segundo a evolução da atividade da doença inflamatória intestinal, apresentando alteração ou não, durante a gestação.

\begin{tabular}{lrrrrrr}
\hline & Retocolite Ulcerativa & Doença de Crohn & \multicolumn{2}{c}{ Total } \\
Atividade da doença durante a gestação & $\mathbf{n}$ & $\boldsymbol{\%}$ & $\mathbf{n}$ & $\boldsymbol{\%}$ & \multicolumn{1}{c}{ n } & \% \\
\hline Com alteração & 14 & 22,2 & 37 & 48,0 & 51 & 36,4 \\
Sem alteração & 49 & 77,8 & 40 & 52,0 & 89 & 63,6 \\
Total & 63 & 100,0 & 77 & 100,0 & 140 & 100,0 \\
\hline
\end{tabular}

Tabela 3 - Distribuição das doentes, segundo a evolução da atividade da doença inflamatória intestinal durante a gestação.

\begin{tabular}{lrrrrrr}
\hline & \multicolumn{2}{c}{ Retocolite Ulcerativa } & \multicolumn{2}{c}{ Doença de Crohn } & \multicolumn{2}{c}{ Total } \\
& $\mathbf{n}$ & $\mathbf{\%}$ & $\mathbf{N}$ & $\mathbf{\%}$ & n & \% \\
\hline Melhora & 7 & 11,1 & 28 & 36,4 & 35 & 25,0 \\
Inalterado com atividade & 21 & 33,3 & 11 & 14,3 & 33 & 23,6 \\
Inalterado sem atividade & 28 & 44,5 & 29 & 37,6 & 57 & 40,0 \\
Piora & 7 & 11,1 & 9 & 11,7 & 16 & 11,4 \\
Total & 63 & 100,0 & 77 & 100,0 & 140 & 100,0 \\
\hline
\end{tabular}


Rev bras Coloproct Julho/Setembro, 2009 Leonardo Corrêa de Oliveira Rodrigues e Cols.
Quanto à melhora da atividade da RC e DC com e sem medicamento, não houve diferença significativa ( $\mathrm{p}=0,9999 \mathrm{e} \mathrm{p}=0,3930$, respectivamente). Também não foi evidenciada diferença significativa nas taxas de piora entre RC com e sem o uso de medicamentos $(\mathrm{p}=0,9999)$ e entre a DC com e sem medicamentos $(\mathrm{p}=0,9999)$.

As taxas de melhora entre RC e DC sem medicamento tiveram diferença significativa $(p=0,0103)$ e nas pacientes que utilizaram não houve diferença significativa $(\mathrm{p}=0,0570)$. Não houve diferença estatística nas taxas de piora dos grupos de RC e DC com $(\mathrm{p}=0,9999)$ e sem o uso de medicamentos ( $\mathrm{p}=0,9999)$, durante a gestação. Os dados detalhados figuram na tabela 4:

\subsection{Efeitos da DII sobre a gestação}

\subsubsection{Prematuridade}

Houve diferença significante entre as pacientes antes e após o início da doença em relação à prematuridade $\left(x^{2}=6,7205, p=0,009\right)$ conforme demonstrado no Gráfico 1 e abortamento provocado $\left(\mathrm{x}^{2}=\right.$ 5,8466, p=0,01561), no Gráfico 5:

Das 416 gestações antes do início dos sintomas da mãe, 30 nasceram prematuros (7,2\%). Das 140 gestações após o início dos sintomas na mãe, 21 nasceram prematuros.

\subsubsection{Baixo peso ao nascer}

Não foi encontrada diferença significativa quanto ao baixo peso ao nascer $\left(\mathrm{x}^{2}=0,413 / \mathrm{p}=0,5204\right)$ entre os nascidos vivos de mães, antes e depois do início dos sintomas relacionados à DII (Gráfico 2).

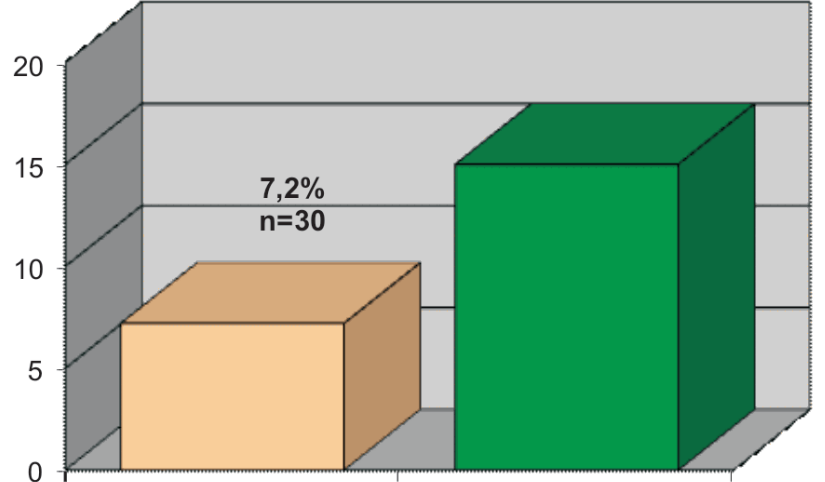

$\square$ ANTES DO INÍCIO DOS SINTOMAS

$\square$ DEPOIS DO INICIO DOS SINTOMAS

$x^{2}=6,7205 / p=0,009$

Gráfico 1 - Taxa de prematuridade, antes e após o início dos sintomas relacionados à DII.

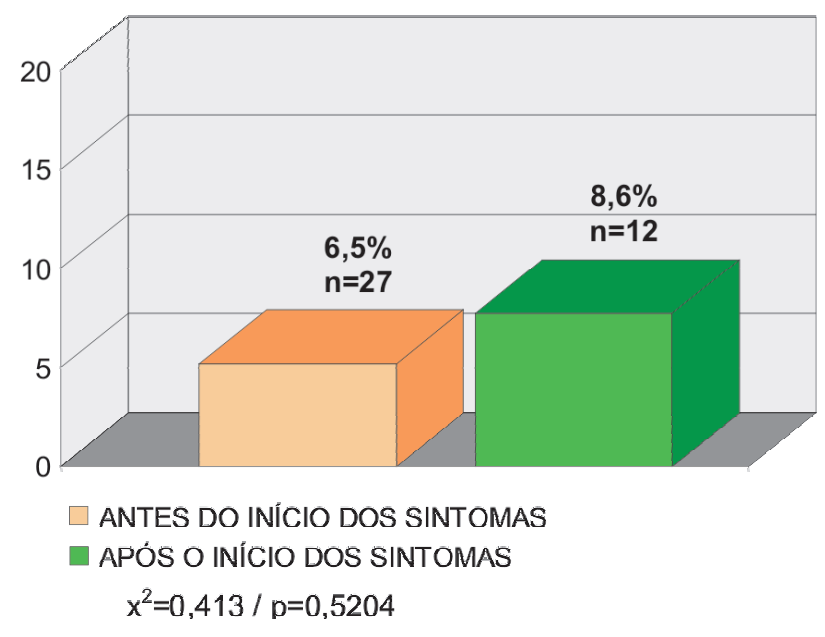

Gráfico 2 - Taxa de baixo peso ao nascer, antes e após o início dos sintomas relacionados à DII.

Tabela 4 - Distribuição das doentes, segundo a evolução da atividade da doença inflamatória intestinal, com ou sem o uso de medicações, durante a gestação.

\begin{tabular}{|c|c|c|c|c|c|c|c|c|c|c|c|c|}
\hline \multirow{3}{*}{ Quadro Clínico } & \multicolumn{4}{|c|}{ Retocolite Ulcerativa } & \multicolumn{4}{|c|}{ Doença de Crohn } & \multicolumn{4}{|c|}{ Total } \\
\hline & \multicolumn{2}{|c|}{$\mathbf{S} / \mathbf{M}^{*}$} & \multicolumn{2}{|c|}{$\mathbf{C} / \mathbf{M}^{\#}$} & \multicolumn{2}{|c|}{$\mathbf{S} / \mathbf{M}$} & \multicolumn{2}{|c|}{$\mathbf{C} / \mathbf{M}$} & \multicolumn{2}{|c|}{$\mathbf{S} / \mathbf{M}$} & \multicolumn{2}{|c|}{$\mathrm{C} / \mathrm{M}$} \\
\hline & $\mathbf{n}$ & $\%$ & $\mathbf{n}$ & $\%$ & $\mathbf{n}$ & $\%$ & $\mathbf{n}$ & $\%$ & $\mathbf{n}$ & $\%$ & $\mathbf{n}$ & $\%$ \\
\hline Melhora & 5 & 10,8 & 2 & 11,8 & 20 & 33,3 & 8 & 47,0 & 25 & 23,6 & 10 & 29,4 \\
\hline Inalterado com atividade & 14 & 30,6 & 7 & 41,1 & 6 & 10,0 & 5 & 29,4 & 20 & 18,9 & 12 & 35,3 \\
\hline Inalterado sem atividade & 22 & 47,8 & 6 & 35,3 & 27 & 45,0 & 2 & 11,8 & 49 & 46,2 & 8 & 23,5 \\
\hline Piora & 5 & 10,8 & 2 & 11,8 & 7 & 11,7 & 2 & 11,8 & 12 & 11,3 & 4 & 11,8 \\
\hline Total & 46 & 100,0 & 17 & 100,0 & 60 & 100,0 & 17 & 100,0 & 106 & 100,0 & 34 & 100,0 \\
\hline
\end{tabular}

* sem medicação para o tratamento da DII durante a gestação.

\# com medicação para o tratamento da DII durante a gestação. 
Rev bras Coloproct Julho/Setembro, 2009 Leonardo Corrêa de Oliveira Rodrigues e Cols.
Das 416 gestações antes do início dos sintomas da mãe, 27 nasceram com baixo peso ao nascer $(6,5 \%)$. Dos 140 que nasceram após o início dos sintomas na mãe, 12 nasceram com baixo peso ao nascer $(8,6 \%)$.

\subsubsection{Anomalias congênitas}

Não foi encontrada diferença significativa quanto às anomalias congênitas (Teste exato de Fisher: $\mathrm{p}=0,6447$ ) entre os nascidos vivos antes e depois do início das DII. Os resultados estão demonstrados no gráfico 3 .

Das gestações antes do início dos sintomas da mãe, 4 nasceram com anomalias congênitas $(1,0 \%)$. Dos 140 que nasceram após o início dos sintomas na mãe, 2 apresentavam anomalias congênitas $(1,4 \%)$.

As quatro anomalias congênitas $(1,0 \%)$ encontradas nos filhos nascidos de mães antes do início dos sintomas da DII foram duas alterações cardíacas, um recém-nascido com pé torto e um com anencefalia. As duas anomalias congênitas $(1,4 \%)$ encontradas nos filhos nascidos de mães, após o início dos sintomas da DII, foram um recém-nascido com alterações cardíacas e outro com ptose renal.

\subsubsection{Natimortos}

Não foi encontrada diferença significativa quanto à natimortalidade, antes e após o início dos sintomas da DII (Teste exato de Fisher: p=0,2245) (Gráfico 4).

Das gestações antes do início dos sintomas da mãe, 9 foram natimortos (2,2\%). Dos 140 que nasceram após o início dos sintomas na mãe, 6 foram natimortos $(4,3 \%)$.

\subsubsection{Abortamento}

Não houve diferença significante quanto ao abortamento natural, entre os grupos antes e após o início dos sintomas da DII $\left(\mathrm{x}^{2}=4.884, \mathrm{p}=0,27\right)$. Todos os abortamentos provocados foram realizados por conduta da paciente e não por indicação terapêutica.

Houve diferença significante quanto ao abortamento provocado, entre os grupos antes e após o início dos sintomas da DII ( $\mathrm{p}=0,003)$, sendo maior antes dos sintomas. Os resultados estão demonstrados no gráfico 5 .

Das gestações antes do início dos sintomas da DII na mãe, houve 50 abortamentos espontâneos $(12,0 \%)$ e 36 abortamentos provocados $(8,6 \%)$. Das 140 gestações após o início dos sintomas da DII na

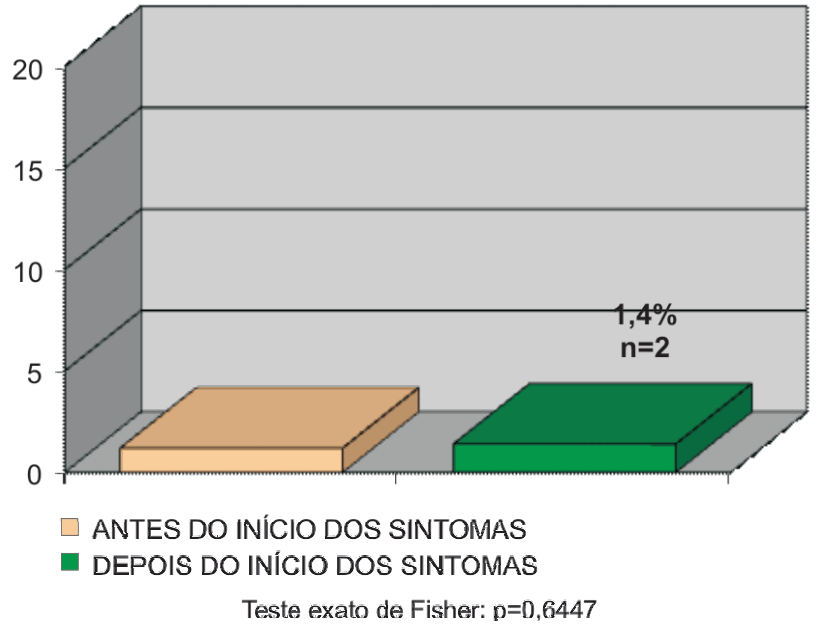

Gráfico 3 - Taxa de anomalias congênitas, antes e após o início dos sintomas relacionados à DII.

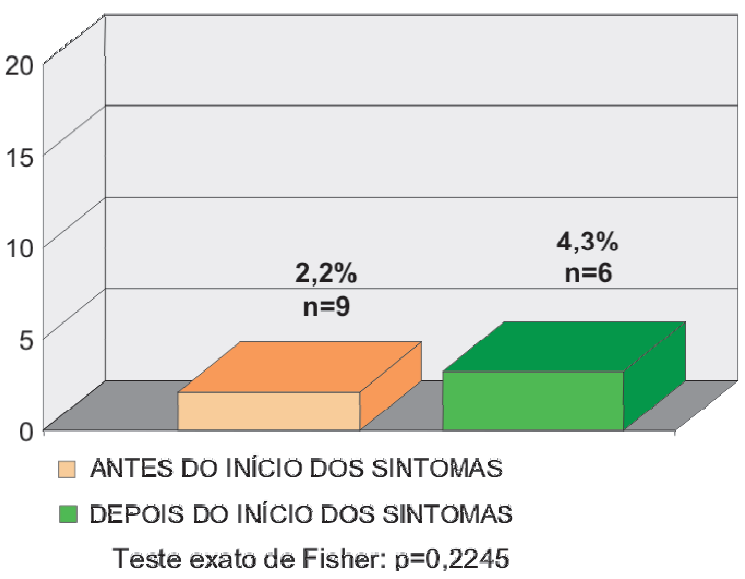

Gráfico 4 - Taxa de natimortos, antes e após o início dos sintomas relacionados à DII.

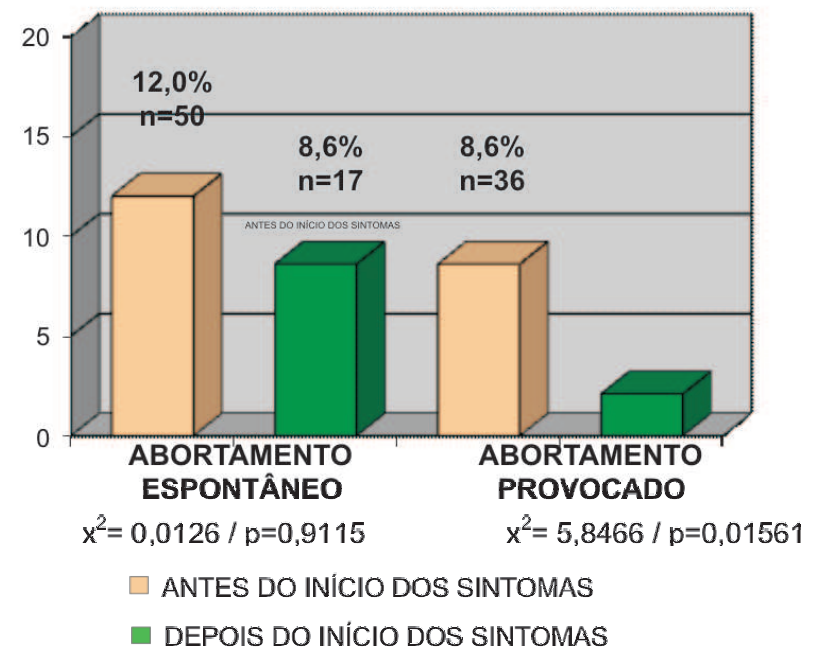

Gráfico 5 - Taxa de abortamento, antes e após o início dos sintomas relacionados à DII. 
Rev bras Coloproct Julho/Setembro, 2009 Leonardo Corrêa de Oliveira Rodrigues e Cols. mãe, houve 17 abortamentos espontâneos $(8,6 \%)$ e 3 abortamentos provocados $(2,1 \%)$.

\subsubsection{Partos normais e cesáreos}

Não houve diferenças significativas, quando comparada a atividade da doença e o tipo de parto, antes e após o início dos sintomas relacionados à DII $\left(\mathrm{x}^{2}=5.525, \mathrm{p}=0,09\right)$. Os resultados estão demonstrados no gráfico 6 .

\subsection{Atividades da RC e DC e as altera- ções do concepto}

Ao total de pacientes com RC, 28 estavam com a doença ativa durante a gestação e 35 inativos. No

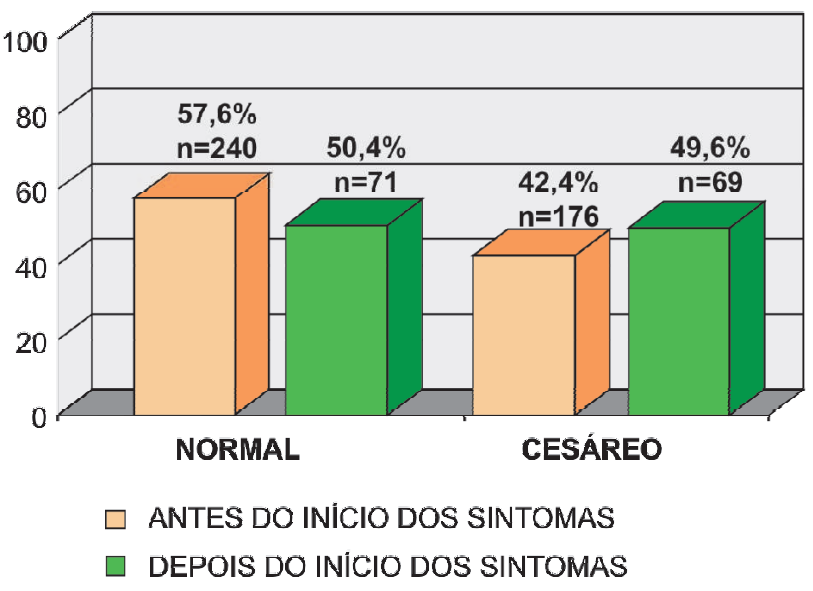

Gráfico 6 - Taxa de partos normais e cesáreos, antes e após o início dos sintomas relacionados à DII. grupo de DC, em 57 pacientes a doença estava ativa e em 20, inativa.

Em relação à prematuridade, não houve diferença significativa entre $\mathrm{RC}$ e DC $(\mathrm{p}=0,1524)$ e entre RC ativo e inativo durante a gestação $(p=0,2138)$. Entretanto na DC houve diferença entre doença ativa e inativa ( $p<0,0001)$.

Como não houve um número representativo de anomalias congênitas nos grupos, torna- se sem sentido a análise estatística.

Em relação às taxas de natimortalidade, não houve diferença significativa entre DC e RC, independente da atividade da doença $(p>0,0649)$.

Em relação ao baixo peso ao nascer, não houve diferença significativa nas taxas de baixo peso entre DC e RC ( $p=0,7680)$, e entre $R C$ ativo e inativo ( $p$ $=0,6844)$. Entretanto, houve diferença entre DC ativo e inativo $(p=0,0365)$.

Em relação à taxa de abortamento natural e provocado, não houve diferença significativa entre DC e RC, independente da atividade da doença $(p>0,1942)$.

As alterações do concepto conforme a atividade da RC e DC, estão distribuídos na tabela 5.

5.5 Localização, atividade da DII e as alterações do concepto

Houve diferença significativa na proporção de prematuridade $(\mathrm{p}=0,018)$, baixo peso ao nascer $(\mathrm{p}=0,018)$ e natimorto $(\mathrm{p}=0,037)$, quando a $\mathrm{RC}$ acometeu todo o cólon. A proporção estimada de prematuridade e baixo peso ao nascer dentre os paci-

Tabela 5 - Distribuição dos filhos de mulheres com DII, segundo as alterações do concepto e a atividade da RC e DC durante a gestação.

\begin{tabular}{|c|c|c|c|c|c|c|c|c|c|}
\hline \multirow[t]{2}{*}{$\begin{array}{l}\text { DII e Alterações } \\
\text { do Concepto }\end{array}$} & \multicolumn{2}{|c|}{$\begin{array}{c}\text { RC } \\
\text { Ativa }\end{array}$} & \multicolumn{2}{|c|}{$\begin{array}{c}\mathbf{R C} \\
\text { Inativa }\end{array}$} & \multicolumn{2}{|c|}{$\begin{array}{c}\text { DC } \\
\text { Ativa }\end{array}$} & \multicolumn{2}{|c|}{$\begin{array}{c}\text { DC } \\
\text { Inativa }\end{array}$} & \multirow{2}{*}{$\begin{array}{r}\text { Total } \\
\mathbf{n} \\
\end{array}$} \\
\hline & $\mathbf{n}$ & $\%$ & $\mathbf{n}$ & $\%$ & $\mathbf{N}$ & $\%$ & $\mathbf{n}$ & $\%$ & \\
\hline Prematuridade & 1 & 1,6 & 5 & 8,0 & 4 & 5,2 & 11 & 14,3 & 21 \\
\hline Anomalia & 1 & 1,6 & - & - & - & - & 3 & 3,9 & 4 \\
\hline Natimorto & - & - & 3 & 4,8 & - & - & 3 & 3,9 & 6 \\
\hline Baixo Peso & 2 & 3,2 & 4 & 6,3 & 2 & 2,6 & 4 & 5,2 & 12 \\
\hline AB*. Natural & 4 & 6,3 & 5 & 8,0 & 4 & 5,2 & 4 & 5,2 & 17 \\
\hline AB*. Provocado & - & - & 1 & 1,6 & 1 & 1,3 & 1 & 1,3 & 3 \\
\hline Parto Normal & 15 & 23,8 & 17 & 26,7 & 18 & 23,4 & 21 & 27,3 & 71 \\
\hline Parto Cesáreo & 15 & 23,8 & 16 & 25,4 & 19 & 24,7 & 19 & 24,7 & 69 \\
\hline
\end{tabular}

*Abortamento. 

Leonardo Corrêa de Oliveira Rodrigues e Cols. entes com comprometimento de todo o cólon foi de 83,33\% [IC 95\%: 10,29\%; 100,00\%]. Todos os natimortos foram do grupo das gestantes com o comprometimento de todo o cólon.

A distribuição das doentes segundo as alterações do concepto e a localização da RC está demonstrada na tabela 6 .

Não houve diferença significativa entre a localização e a atividade da DC (Intestino delgado e outras localizações: $p=0,8893$; intestino grosso e outras localizações: $p=0,9999$; Perianal e outras localizações: $\mathrm{p}=0,7578$; Intestino delgado e grosso e outras localizações: $p=0,8813$; Intestino delgado e perianal e outras localizações: $p=0,6513$; Intestino grosso e perianal: sem diferença entre proporções: $p=0,8123$ ).

A distribuição das doentes segundo as alterações do concepto e a localização da DC está demonstrada na tabela 7 .
Houve uma maior incidência de parto cesáreo quando a DC acometeu a região perianal e o intestino grosso concomitantemente $\left(\mathrm{x}^{2}=19.763, \mathrm{p}=0,004\right)$. Não houve diferença estatística entre os tipos de parto, quando a doença acomete a região perianal isoladamente ou outras regiões $\left(x^{2}=9.698, p=0,098\right)$.

\subsection{O uso de medicamentos e as altera- ções do concepto}

Os medicamentos mais utilizados durante o período gestacional foram os salicilatos e os corticosteroides. Os outros medicamentos como os imunossupressores, antibióticos e anticorpos monoclonais não foram utilizados de forma expressiva. Apenas em três gestações houve o uso de imunossupressores, em três, ciprofloxacino, em duas, metronidazol e em duas pacientes haviam recebido infliximabe até dois meses antes da concepção.

Tabela 6 - Distribuição das alterações do concepto e o local da inflamação nas mães com RC durante a gestação.

\begin{tabular}{|c|c|c|c|c|c|c|c|c|c|c|}
\hline \multirow{2}{*}{$\begin{array}{l}\text { Alterações do Concepto } \\
\text { Local da Inflamação }\end{array}$} & \multicolumn{2}{|c|}{ Prematuridade } & \multicolumn{2}{|c|}{$\begin{array}{l}\text { Baixo } \\
\text { Peso }\end{array}$} & \multicolumn{2}{|c|}{$\begin{array}{c}\text { Anomalias } \\
\text { Congênitas }\end{array}$} & \multicolumn{2}{|c|}{ Natimortos } & \multicolumn{2}{|c|}{$\begin{array}{c}\text { Abortamento } \\
\text { Natural }\end{array}$} \\
\hline & $\mathbf{n}$ & $\%$ & $\mathbf{n}$ & $\%$ & $\mathbf{n}$ & $\%$ & $\mathbf{n}$ & $\%$ & $\mathbf{n}$ & $\%$ \\
\hline Porção Distal c & - & - & - & - & & - & - & - & - & - \\
\hline Hemicólon Esquerdo & 1 & 1,6 & 1 & 1,6 & - & - & - & - & 4 & 6,3 \\
\hline Todo o Cólon & 5 & 8,0 & 5 & 8,0 & 1 & 1,6 & 3 & 4,8 & 5 & 8,0 \\
\hline Total & 6 & 9,6 & 6 & 9,6 & 1 & 1,6 & 3 & 4,8 & 9 & 14,3 \\
\hline
\end{tabular}

Tabela 7 - Distribuição das alterações do concepto e o local da inflamação nas mães com DC durante a gestação.

\begin{tabular}{|c|c|c|c|c|c|c|c|c|c|c|}
\hline \multirow{2}{*}{$\begin{array}{l}\text { Alterações do Concepto } \\
\text { Local da Inflamação }\end{array}$} & \multicolumn{2}{|c|}{ Prematuridade } & \multicolumn{2}{|c|}{$\begin{array}{l}\text { Baixo } \\
\text { Peso }\end{array}$} & \multicolumn{2}{|c|}{$\begin{array}{l}\text { Anomalias } \\
\text { Congênitas }\end{array}$} & \multicolumn{2}{|c|}{ Natimortos } & \multicolumn{2}{|c|}{$\begin{array}{c}\text { Abortamento } \\
\text { Natural }\end{array}$} \\
\hline & $\mathbf{n}$ & $\%$ & $\mathbf{n}$ & $\%$ & $\mathbf{n}$ & $\%$ & $\mathbf{n}$ & $\%$ & $\mathbf{n}$ & $\%$ \\
\hline Intestino Delgado & 3 & 3,9 & 1 & 1,3 & 1 & 1,3 & - & - & 1 & 1,3 \\
\hline Intestino Grosso & - & - & - & - & - & - & - & - & - & - \\
\hline Região Perianal & 1 & 1,3 & 1 & 1,3 & - & - & 1 & 1,3 & - & - \\
\hline Intestino Delgado e Grosso & 3 & 3,9 & 1 & 1,3 & 1 & 1,3 & - & - & 2 & 2,6 \\
\hline Intestino Delgado e Região Periana & al & 3,9 & 1 & 1,3 & - & - & 1 & 1,3 & 1 & 1,3 \\
\hline $\begin{array}{l}\text { Intestino Grosso e Região Perianal } \\
\text { Intestino Delgado e Grosso e }\end{array}$ & 12 & 2,6 & 1 & 1,3 & - & - & - & - & - & - \\
\hline Região Perianal & 3 & 3,9 & 1 & 1,3 & 1 & 1,3 & 1 & 1,3 & 2 & 2,6 \\
\hline Total & 15 & 16,9 & 6 & 6,5 & - & - & 3 & 3,9 & 8 & 10,4 \\
\hline
\end{tabular}


Rev bras Coloproct Julho/Setembro, 2009 Leonardo Corrêa de Oliveira Rodrigues e Cols.
Foram administrados salicilatos durante 21 gestações. Não houve diferença significativa entre o uso ou não da medicação quanto à presença de alterações no concepto $\left(\mathrm{x}^{2}=2.451, \mathrm{p}=0,17\right)$. As alterações e a evolução do concepto de mães que utilizaram salicilatos durante a gestação estão demonstradas no gráfico 7.

Foram administrados corticosteroides durante 15 gestações. Não houve diferença significativa entre o uso ou não da medicação quanto à presença de alterações no concepto $\left(\mathrm{x}^{2}=3.714, \mathrm{p}=0,14\right)$ (prematuridade $(\mathrm{p}=0,9999)$; anomalias congênitas $(\mathrm{p}=0,3005)$; baixo peso ao nascer $(\mathrm{p}=1585)$; natimortalidade $(\mathrm{p}=0,4344)$ e aborto espontâneo $(\mathrm{p}=0,8880)$. As alterações e a evolução do concepto de mães que utilizaram corticosteroides durante a gestação estão demonstradas no gráfico 8 .

As alterações e a evolução do concepto de mães que não utilizaram medicamentos durante a gestação estão demonstradas no gráfico 9.

Dos 69 pacientes que utilizavam alguma medicação para o tratamento da DII, antes do período gestacional, $19(27,5 \%)$ pararam de utilizar medicações, durante a gravidez porque estavam bem em relação à doença, 8 pacientes $(11,6 \%)$ pararam por orientação médica e $5(7,2 \%)$ pacientes pararam por medo dos efeitos colaterais.

\section{DISCUSSÃO}

A DII é uma moléstia que atinge principalmente jovens, sendo diagnosticada, na sua grande maioria, na $2^{\mathrm{a}}$ e $3^{\mathrm{a}}$ década de vida. Tem- se verificado uma incidência crescente desta doença nos últimos anos, em vários países [34-36]. Em nosso meio, o grande número de trabalhos publicados corrobora com esse aumento de incidência [37-50]. Como a maioria dos pacientes é jovem, sendo aproximadamente $50 \%$ do sexo feminino, há interesse em saber como a DII atua sobre a gestação e o feto e vice- versa. Por essa razão julgamos oportuno este estudo. Outro aspecto que nos estimulou a estudar este tema, foram os achados controversos da literatura.

\subsection{Fecundidade}

Comparando a média de gestações por paciente com DII, em relação aos dados fornecidos pelo Datasus [30], verificamos que os dados foram similares ao do restante da população no Brasil, nos últimos cinco anos. Portanto a DII, não interferiu neste índice.

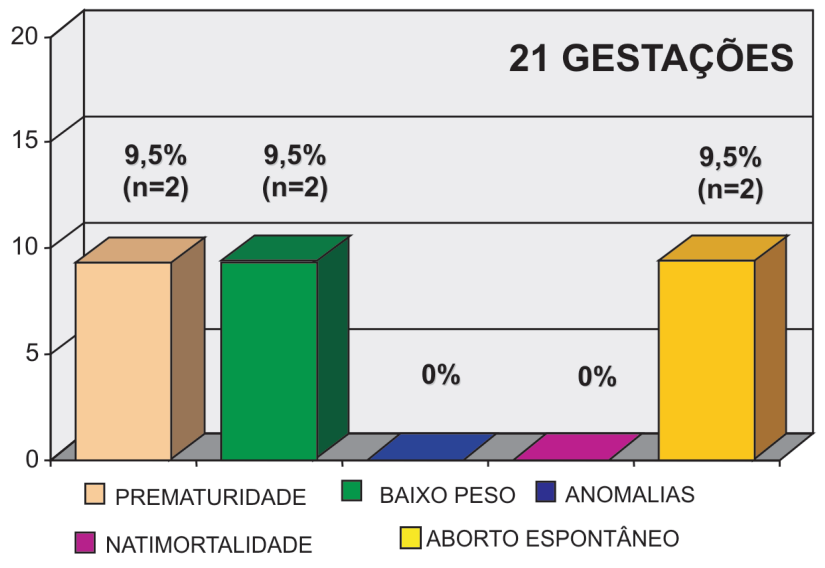

Gráfico 7 - Alterações e evolução do concepto com o uso de salicilatos durante a gestação pela mãe.

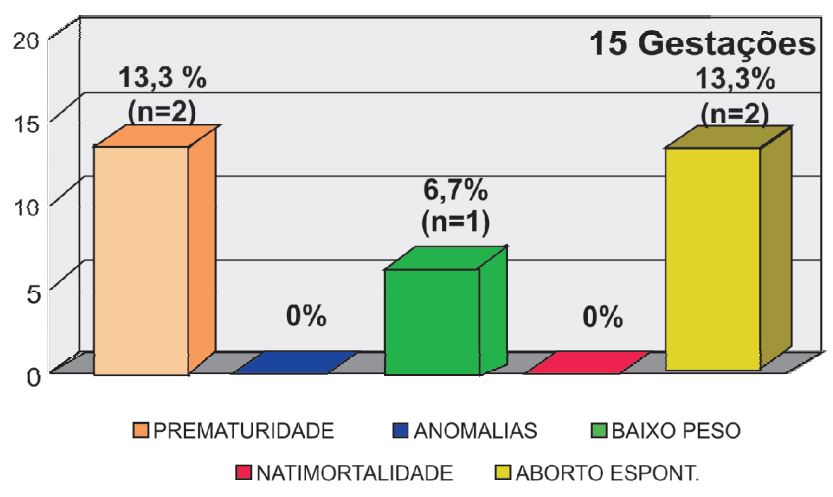

Gráfico 8 - Alterações e evolução do concepto com o uso de corticosteróides durante a gestação pela mãe.

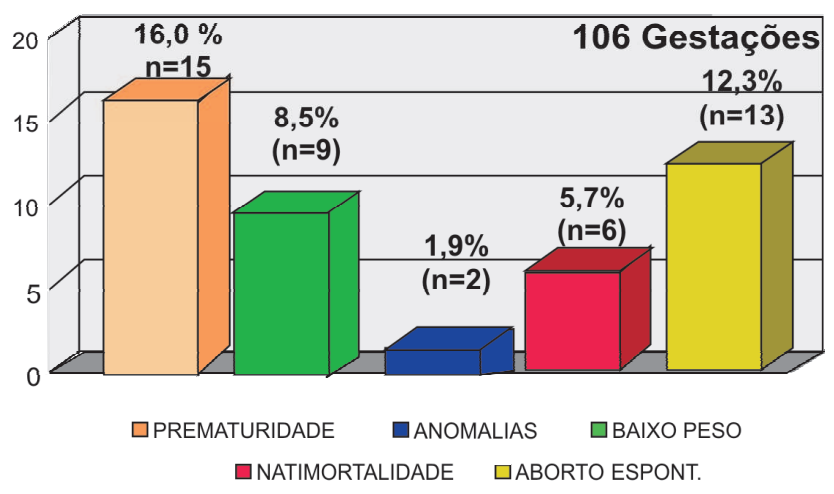

Gráfico 9 - Alterações e evolução do concepto sem o uso de medicações durante a gestação pela mãe.

Dados similares foram obtidos por outros autores [51]. Embora a média de gestações por paciente tenha se mantido, verificamos que após o início da DII, houve uma diminuição não significativa da média das gesta- 
ções. Entre os motivos possíveis para justificar essa diminuição, devemos considerar as seguintes possibilidades:

1. Um grupo de pacientes já havia constituído família e não desejava mais filhos, ou não tinha companheiro $(53,8 \%)$.

2. Outro grupo não desejava filhos por vários motivos não relacionados à DII (problemas conjugais, excesso de trabalho, etc.)

3. E, finalmente, um terceiro grupo responsabilizava a doença como causa de não ter filhos, representando aproximadamente um quinto desta casuística $(20,6 \%)$. No entanto é importante frisar que o fato de responsabilizar a doença, não é sinônimo a impossibilidade de concepção. Este dado, embora não o motivo mais frequente para não engravidar, demonstra a importância das DII no planejamento familiar. Outra consequência secundária deste fato é a eventual influência da DII no relacionamento conjugal, uma vez que pode frustrar a expectativa do cônjuge em formar uma família.

As causas mais prováveis para os casais não terem filhos são a dificuldade de relacionamento, problemas com a imagem corporal, medo de engravidar e aconselhamento médico inapropriado, conforme apontado por outros autores [52-54].

Em nossa casuística, houve redução do número de filhos de mães com DII por escolha própria, quer por medo da doença ou simplesmente por não quererem ter filhos. Portanto, trata-se na maioria das vezes da escolha da paciente e não a impossibilidade de conceber conforme já referido na literatura por diversos autores [7,24,52-54].

Estudos antes da década de 90 demonstraram altas taxas de infertilidade, atingindo até $66 \%$ das mulheres com DC [55,56] e até $49 \%$ das mulheres com $\mathrm{RC}$ [3]. Esta taxa diminuiu nos estudos mais recentes oscilando entre 5 e $14 \%$ e não se diferencia da taxa da população geral, o mesmo fato foi por nós observado $[19,20,24,30,54]$. Provavelmente os trabalhos mais recentes incluem pacientes tratadas com drogas inexistentes até então e maiores cuidados com a desnutrição o que justificaria a discrepância entre os dados.

A infertilidade aumenta com a idade [8], cirurgias prévias e outras alterações principalmente na DC grave, quando há comprometimento ovariano e da tuba uterina $[7,17,54]$. Mulheres que desenvolvem DII antes da primeira gestação têm menos filhos do que a população controle ou mulheres que desenvolveram a doença após ter tido seu primeiro filho [17]. Embora exista a probabilidade de terem menos filhos por razões anatômicas não se podem excluir outras razões como o medo da doença, recomendações médicas, entre outras.

Poucas pacientes não conseguiram engravidar. Porém, não foi objetivo deste trabalho averiguar as causas, que poderiam inclusive ser consequentes aos problemas relacionados ao cônjuge. Portanto, nossos dados não nos permitem concluir nada a respeito da fertilidade.

\subsection{Efeitos da gestação sobre a DII}

A grande maioria das mulheres com DII (aproximadamente 2/3) não apresentou alteração da atividade da doença quando ficaram grávidas.

No entanto, quando estudamos isoladamente a DC e a RC, verificamos que o grupo constituído por pacientes com RC tendeu a se manter sem alteração do quadro clínico em porcentagem superior ao grupo com DC. Nas pacientes com DC, os resultados foram similares entre o grupo que mostrou alteração do quadro clínico com o grupo que permaneceu inalterado durante a gestação. Verificamos ainda que houve tendência maior na DC a apresentar melhora do quadro clínico durante a gestação. Estes dados são coincidentes com os da literatura [3-7,9,11,12,14,18,20,57-63]. Quando avaliamos os grupos que não utilizaram medicações durante a gestação também observamos que as pacientes com DC tiveram uma taxa de melhora da atividade da doença durante esse período maior que no grupo de RC.

Neste estudo, 30\% (3/9) das pacientes que estavam em remissão antes da gestação e que pararam de fazer uso das medicações específicas para o tratamento da DII, durante a gravidez, apresentou piora da atividade da doença. Esta piora é vista quando há a suspensão dos medicamentos de manutenção [10].

\subsection{Efeitos da DII sobre a gestação}

O efeito da DII na gestação e seu prognóstico estão primariamente dependentes da atividade da doença no momento da concepção e durante a gestação. A maioria dos estudos demonstrou que mulheres com doença inativa durante a gestação não apresentou risco maior de prematuridade, anomalias congênitas, natimortalidade, baixo peso ao nascer e abortamento espontâneo [6-11,13-15,64]. Quando a doença está ativa 
no momento da concepção ou durante a gestação há associação de pior prognóstico da gestação e dos conceptos em alguns estudos [5,13,15,19,20,21]. Entretanto, existem estudos que demonstraram que a DII é um fator de risco independente para as complicações do feto independente da atividade da doença e tratamento clínico [65]. Esse risco parece ser maior na DC do que na RC [18].

Estudos caso-controles demonstraram risco maior de prematuridade, baixo peso ao nascer e abortamento espontâneo em conceptos de mães com DC, principalmente após o diagnóstico da doença [5,22,23], exceto quanto às anomalias congênitas [66] e natimortalidade [9], que não foram maiores.

No nosso estudo, observou-se maior taxa de prematuridade nas gestantes com DC sem atividade do que naquelas com atividade.

Embora o número de partos cesáreos tenha sido maior na população do estado de São Paulo, as pacientes que, aqui no Hospital das Clínicas de São Paulo foram tratadas, provavelmente tiveram um melhor atendimento obstétrico dando maior incentivo ao parto normal, exceto nos casos em que a DC tenha acometido a região perianal.

Realizamos um levantamento dos dados relacionados com o prognóstico dos fetos e tipos de partos em gestação de mulheres do Estado de São Paulo no período de 2000 a 2005, para comparação com os dados do nosso trabalho. Estes dados figuram na tabela 8 .

Pode-se observar que não houve alteração significativa entre os valores de cada categoria, ao longo dos 6 anos, fornecidos pelo Datasus, mostrando uma uniformidade dos dados.
Embora o número total tenha sido similar ao obtido pelo Datasus do Estado de São Paulo, quando analisamos os números antes e depois da DII, observamos que houve incidência significativamente maior de prematuridade após o início da doença.

A comparação das alterações e evolução do concepto neste estudo e nos conceptos do estado de São Paulo nos anos de 2000 a 2005 estão demonstradas no gráfico 10.

Fizemos um teste qui-quadrado de contingência, associando as alterações do concepto e os tipos de parto no estudo e no Datasus. Não foram encontradas diferenças significativas, quanto à prematuridade $\left(\mathrm{X}^{2}=1,728, \mathrm{p}=0,62\right)$, às anomalias congênitas $\left(\mathrm{X}^{2}=6,321\right.$, $\mathrm{p}=0,32)$, à natimortalidade $\left(\mathrm{X}^{2}=5,684, \mathrm{p}=0,47\right)$, ao baixo peso $\left(X^{2}=0,994, p=0,91\right)$. Foi encontrada diferença significativa apenas para o tipo de parto $\left(X^{2}=13,623\right.$, $\mathrm{p}=0,038)$. Nos dados do Datasus o parto cesáreo foi mais frequente em média.

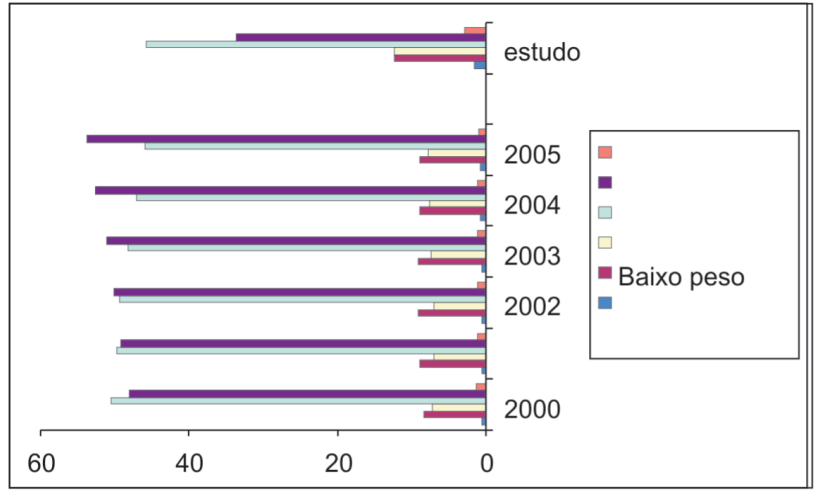

Gráfico 10 - Alterações e evolução do concepto neste estudo e nos conceptos do estado de São Paulo nos anos de 2000 a 2005.

Tabela 8 - Distribuição segundo o prognóstico dos fetos e tipos de partos em gestação de mulheres do Estado de São Paulo no período de 2000 a 2005.

\begin{tabular}{lrrrrrr}
\hline $\begin{array}{l}\text { Alterações do concepto e } \\
\text { Tipo de parto }\end{array}$ & $\mathbf{2 0 0 0}$ & $\mathbf{2 0 0 1}$ & $\mathbf{2 0 0 2}$ & $\mathbf{2 0 0 3}$ & $\mathbf{2 0 0 4}$ & $\mathbf{2 0 0 5}$ \\
\hline Prematuridade & $7,1 \%$ & $7,0 \%$ & $7,0 \%$ & $7,3 \%$ & $7,6 \%$ & $7,7 \%$ \\
Baixo peso ao nascer & $8,4 \%$ & $8,9 \%$ & $9,0 \%$ & $9,2 \%$ & $9,0 \%$ & $8,9 \%$ \\
Anomalia congênita & $0,6 \%$ & $0,6 \%$ & $0,6 \%$ & $0,6 \%$ & $0,7 \%$ & $0,7 \%$ \\
Natimortos & $1,3 \%$ & $1,2 \%$ & $1,2 \%$ & $1,0 \%$ & $1,0 \%$ & $0,9 \%$ \\
Parto vaginal & $51,2 \%$ & $50,3 \%$ & $49,6 \%$ & $48,6 \%$ & $47,2 \%$ & $46,1 \%$ \\
Parto cesáreo & $48,8 \%$ & $49,7 \%$ & $50,4 \%$ & $51,4 \%$ & $52,8 \%$ & $53,9 \%$ \\
\hline
\end{tabular}

Fonte: Datasus. 


\section{concepto}

6.4 Localização da DII e as alterações do

Não encontramos incidência maior de alterações do concepto nas pacientes com atividade da doença, quanto à localização da mesma, assim como outros autores $[17,65,66]$. No entanto, há divergência na literatura quanto a este aspecto [66]. O acometimento da porção ileal na DC pode estar relacionado à recémnascidos com baixo peso ao nascer [67]. Apenas foi observada taxa maior de partos cesáreos nas pacientes com DC e acometimento da região perianal e intestino grosso, concomitantemente, pelo provável risco de piora da lesão perianal, com o parto vaginal. A cirurgia prévia à gestação pode ser um preditor para recém-nascidos com baixo peso, porém é uma afirmação conflitante [14,67].

\subsection{Medicamentos utilizados na DII du- rante a gestação}

A maioria das drogas utilizadas na doença inflamatória intestinal é segura quando utilizada durante a gestação. Porém muitas mulheres ficam apreensivas em utilizá-las durante a gestação e optam por descontinuar o tratamento, mesmo contra a orientação médica. Há situações em que há a descontinuidade do tratamento por orientação médica, sob a justificativa que as medicações poderiam prejudicar o concepto. O tratamento, seu início ou continuação da terapêutica medicamentosa, deve ser discutido com a paciente, devendo-se interromper o uso quando a doença estiver quiescente ou manter em doses efetivas menores. O importante é esclarecer à paciente que doença ativa sem medicação é prejudicial ao concepto $[7,13]$. As drogas devem ser utilizadas em sua dose efetiva mais baixa e em um menor tempo possível.

Neste estudo, as únicas medicações estudadas foram os aminossalicilatos e o corticosteroides, pois o uso das outras medicações durante a gestação não foram em número expressivo ou significativo para se submeter à análise estatística.

Observamos algumas características nas pacientes com DII, quando analisamos a atividade da doença durante a gestação com e sem o uso de medicamentos específicos para seu tratamento. As taxas de piora da atividade da doença, quando as pacientes com RC e DC ficaram grávidas, foram similares, com ou sem o uso de medicações durante a gestação, para o tratamento da DII.
As pacientes com DC apresentaram taxa de melhora da atividade da doença maior do que as pacientes com RC, independente do uso de medicações. Porém, o grupo de pacientes com DC que utilizou medicamentos durante a gestação teve taxa de melhora semelhante comparada ao grupo de pacientes que não utilizou medicamentos.

A taxa de pacientes que utilizaram medicamentos, mesmo sem atividade da doença durante a gestação, foi maior no grupo de pacientes com RC. Isso demonstra que mesmo sem atividade da doença antes da gestação, há maior necessidade do uso de medicações durante a gestação na $\mathrm{RC}$, para que se mantenha a doença em remissão durante o período gestacional.

\subsubsection{Aminossalicilatos e DII}

No nosso estudo, não houve maior alterações nos conceptos, quando comparamos o grupo de gestantes que utilizou aminossalicilatos e o que não utilizou nenhuma medicação.

Há alguns trabalhos que demonstraram aumento nas taxas de prematuridade e baixo peso ao nascer naquelas que utilizaram aminossalicilatos, porém esse aumento foi atribuído ao efeito da atividade da doença e não à droga. Ainda não se tem dados suficientes que provem a segurança do uso de altas doses de aminossalicilatos durante a gestação $(>3 \mathrm{~g} / \mathrm{dia})[68,69]$.

Portanto, o uso da sulfassalazina e da mesalazina é considerado seguro durante a gestação [7], sendo recomendado o uso de até $2-3 \mathrm{~g} / \mathrm{dia}$.

\subsubsection{Corticosteroides e DII}

No nosso estudo, não houve maior alterações nos conceptos, quando comparamos o grupo de gestantes que utilizou corticosteroides e o que não utilizou nenhuma medicação.

Há alguns estudos que demonstraram risco aumentado de fenda palatina e lábio leporino com o uso de corticosteroides durante a gestação [70-72], além de outros que demonstraram um aumento na incidência de fetos com baixo peso.

\section{CONCLUSÃO}

A DII não influenciou diretamente na fecundidade na grande maioria das pacientes, uma vez que houve predominância de fatores de natureza pessoal. 
Rev bras Coloproct Julho/Setembro, 2009
Interação da Gestação na Atividade da Doença Inflamatória Intestinal e sua Influência sobre o Prognóstico Gestacional e na Fecundidade Leonardo Corrêa de Oliveira Rodrigues e Cols.
Vol. 29
A gestação influenciou positivamente a evolução da DC, tanto nas pacientes que utilizaram medicamentos, quanto nas pacientes que não utilizaram. Já na RC, não houve influência importante da gestação na atividade da doença, uma vez que a grande maioria das gestantes permaneceu com a atividade inalterada, independente do uso ou não da medicação.

A DC interferiu na taxa de prematuridade, mesmo estando inativa durante a gestação, sendo maior após o início dos sintomas relacionados à essa DII, independente da atividade da doença.
Não houve influência da DII sobre o baixo peso ao nascer, anomalias congênitas ou natimortalidade dos conceptos, abortamentos naturais e provocados.

Em relação à localização da doença, houve maior taxa de proles com alterações quando todo o cólon estava comprometido na mãe com RC. Em relação à DC, não houve relação entre a localização da doença e as alterações do concepto.

A DII não influenciou o tipo de parto, exceto nos casos de doença perianal extensa associada à doença colônica.

ABSTRACT: INTRODUCTION: Most of women that develops inflammatory bowel disease (IBD) are in fertile age, concerning doctors and patients to understand this interaction. We evaluated the influence of IBD on fecundity and pregnancy and viceversa. METHODS: the protocols of patients with Crohn's disease (CD) and ulcerative colitis (UC), from 1984 and 2006 , in fertile age, followed at the outpatient clinic were reviewed. Patients were interviewed by the research medical doctor, to complete missing data not found in their protocols. Patients with others colitis, incomplete investigation, not in fertile age or without cognitive capacity were excluded from this study. Preterm delivery, low birth weight, congenital anomalies, stillbirth, miscarriages, types of delivery, disease topography in pregnant patients and drug administration during pregnancy were investigated. The statistic method adopted was the chi-square and Fisher test, with significance level of $5 \%$. No patient refused to participate in this study. RESULTS: 140 pregnancies in 104 patients with IBD were evaluated (UC in 63 and CD in 77 pregnancies). : a reduction of 41.6\% in fecundity was observed after beginning of symptoms related to IBD, with influence of the disease in $20.6 \%(10.3 \%$ of patients did not want to have children because of fear related to disease; $6.5 \%$ because of medical orientation and $2.2 \%$ for poor medical conditions). There was no difference between CD and UC. Most of patients did not want to become pregnant because they already had children, were "underage" or "alone" $(53.3 \%)$ Most of pregnancies did not altered clinic conditions in UC patients (77.8\% / $\mathrm{p}=\mathbf{0 . 0 0 3}$ ). Clinical conditions improved during pregnancies more in CD patients than UC patients ( $\mathrm{p}=0.0007)$. The incidence of preterm delivery, low birth weight and stillbirth was higher when the whole colon was affected in $\mathrm{UC}$ ( $p<0.037$ ). The estimated rate of preterm delivery low birth weight was $83.3 \%$ [IC 95\%: $10.29 \% ; 100.00 \%$ ]. There was no statistic difference between the disease topography and the fetus alteration in $\mathrm{CD}(\mathrm{p} \geq \mathbf{0 . 6 5 1 3})$. In twenty-one and fifteen pregnant women, aminosalicilates and corticosteroids were administered, respectively. In 106 pregnancies, no drugs were administered. There were no higher rates of fetus alteration when aminosalicilates or corticosteroids were administered to mothers with IBD ( $p \geq 0.17$ and $p \geq 0.1585$, respectively). Conclusions: IBD didn't have any direct influence on fecundity in most of the patients. Pregnancy influenced positively on CD evolution, independently of drug use. The preterm birth rate was higher in children of mothers with CD. There were higher rates of fetus alteration when the whole colon was affected in mothers with UC. CD influenced the type of delivery only when perianal disease was associated to colonic disease.

Key words: 1.Proctocolitis 2.Crohn disease 3.Pregnancy 4.Intestinal diseases 5.Fertility.

\section{REFERÊNCIAS}

1. Peppercorn MA. Fertility, pregnancy and nursing in inflammatory bowel disease. Disponível em: http:// www.uptodate.com.

2. Rogers RG, Katz VL. Course of Crohn's disease during pregnancy and its effect on pregnancy outcome: a retrospective review. Am J Perinatol 1995; 12: 262-264.

3. De Dombal FT, Watts JM, Watkinson G, Goligher JC. Ulcerative colitis and pregnancy. Lancet II 1965 Sep 25; 2(7413): 599-602.
4. Mogadam DM, Korelitz BI, Ahmed SW, et al. The course of inflammatory bowel disease during pregnancy and postpartum. Am J Gastroenterology 1981 Apr; 75(4): 265-269.

5. Nielsen $\mathrm{OH}$, Andreason $\mathrm{B}$, Bondensen $\mathrm{S}$, et al. Pregnancy in Crohn's disease. Scand J Gastroenterol 1984 Sep; 19(6): 724732.

6. Lamah M, Scott HJ. Inflammatory bowel disease and pregnancy. Int J Colorectal Dis 2002; 17: 216-22.

7. Caprilli R, Gassull MA, Escher JC, et al. European Crohn's and Colitis Organisation. European evidence based consensus on the diagnosis and management of Crohn's disease: special situations. Gut 2006; 55(Suppl. 1): i36-58. 
Rev bras Coloproct Julho/Setembro, 2009
Interação da Gestação na Atividade da Doença Inflamatória Intestinal e sua Influência sobre o Prognóstico Gestacional e na Fecundidade Leonardo Corrêa de Oliveira Rodrigues e Cols.
Vol. 29
8. Elbaz G, Fich A, Levy A, Holcberg G, Sheiner E. Inflammatory bowel disease and preterm delivery. Int J Gynaecol Obstet 2005; 90: 193-197.

9. Calderwood AH, Kane SV. IBD and pregnancy. Mèd Gen Mèd 2004 Oct 8; 6(4): 14.

10. Beniada A, Benoist G, Maurel J, Dreyfus M. Inflammatory bowel disease and pregnancy: report of 76 cases and review of the literature. J Gynecol Obstet Biol Reprod 2005; 34: 581588 .

11. Ferrero S, Ragni N. Inflammatory bowel disease: management issues during pregnancy. Arch Gynaecol Obstet 2004; 270: 79-85.

12. Miller JP. Inflammatory disease in pregnancy: a review. J R Soc Med 1986; 79: 221-225.

13. Asimakopoulos G. Pregnancy and inflammatory bowel disease. Rev Med Chir Soc Med Nat Iasl 2004; 108: 46- 50.

14. Bush MC, Patel S, Lapinski RH, Stone JL. Perinatal outcomes in inflammatory bowel disease. J Matern Foetal Neonatal Med 2004; 15: 237-241.

15. Woolfson K, Cohen Z, McLeod RS. Crohn's disease and pregnancy. Dis Colon Rectum 1990; 33: 869-873.

16. Bortoli A, Saibeni S, Tatarella M, Prada A, Beretta L, Rivolta R, Politi P, Ravelli P, Imperiali G, Colombo E, Pera A, Daperno M, Carnovali M, Franchis R, Vecchi M on behalf of the Study Group for Inflammatory Bowel Diseases (GSMII). Pregnancy before and after the diagnosis of inflammatory beowel diseases: retrospective case- control study. J Gastroenterol Hepatol 2007; 22: 542-549.

17. Mayberry JF, Weterman IT. European survey of fertility and pregnancy in women with Crohn's disease: a case control study by European collaborative group. Gut 1986; 27:821825 .

18. Baiocco PJ, Korelitz BI. The influence of inflammatory bowel disease and its treatment on pregnancy and foetal outcome. $\mathrm{J}$ Clin Gastroenterol 1984; 6: 211-216.

19. Willoughby CP, Truelove SC. Ulcerative colitis and pregnancy. Gut 1980 Jun; 21: 469-474.

20. Khosla R, Willoughby CP, Jewell DP. Crohn's disease and pregnancy. Gut 1984; 25: 52-56.

21. Schofield PF, Turnbull RB, Hawk WA. Crohn's disease and pregnancy. BMJ 1970 May 9; 2(5705): 364.

22. Dominitz JA, Young JC, Boyko EJ. Outcomes of infants born to mothers with inflammatory bowel disease: a populationbased cohort study. Am J Gastroenterol 2002; 97: 641-648.

23. Fonager K, Sorensen HT, Olsen J, Dahlerup JF, Rasmussen SN. Pregnancy outcome for women with Crohn's disease: a follow-up study based on linkage between national registries. Am J Gastroenterol 1998; 93: 2426-2430.

24. Baird DD, Narendranathan M, Sandler RS. Increased risk of preterm birth for women with inflammatory bowel disease. Gastroenterology 1990; 99: 987-994.

25. Lene Riis, Ida Vind, Patrizia Politi, et al. On behalf of the European Collaborative study group on inflammatory bowel disease (EC-IBD). Does pregnancy change the disease course? A Study in a European Cohort of Patients with Inflammatory Bowel Disease. Am J Gastroenterol 2006; 101: 1539-1545.

26. Srinivasan R. Infliximab treatment and pregnancy outcome in active Crohn's disease. Am J Gastroenterology 2001 Jul; 96(7): 2274-2275.

27. Lichtenstein G, Cohen RD, Feagan BG, et al. Safety of infliximab in Crohn's disease: data from the 5000- patient TREAT registry.Gastroenterology 2004; 126(suppl): A54

28. Katz JA, Antoni C, Keenan GF, et al. Outcome of pregnancy in women receiving infliximab for the treatment of Crohn's disease and rheumatoid arthritis. Am J Gastroenterology 2004 Dec; 99(12): 2385-2392.

29. Rezende J. Obstetrícia. $9^{a}$ edição. Rio de Janeiro: Guanabara Koogan; 2002.

30. Datasus: www.datasus.org.br

31. Truelove SC, Witts LJ. Cortisone in ulcerative colitis: final report on a therapeutic trial. BMJ 1955;2:1041-8.

32. Harvey RF, Bradshaw JM. A simple index of Crohn's disease activity. Lancet 1980;1:514-515.

33. Moretin, Pedro. Estatística Básica. Ed. Atlas, 2005.

34. Sandler R, Loftus E Jr. Epidemiology of inflammatory bowel disease. Em: Sartor R, Sandborn W, editors. Kirsner's inflammatory bowel diseases. $6^{\mathrm{a}}$ edição. Philadelphia: Saunders; 2004. p. 245-62.

35. Epidemiology of inflammatory bowel disease in Asia. Indian $\mathbf{J}$ Gastroenterol 2007 Nov-Dec; 26(6):285-289.

36. Recent trends in the epidemiology of inflammatory bowel diseases: up or down? World J Gastroenterol 2006 Oct; 12(38): 6102-6108.

37. Reis Neto JA, Reis Jr JA. Formas clínicas de apresentação dadoença de Crohn. In:Habr-Gama A, Barone B. Atualização emcoloproctologia. São Paulo: 1995.

38. Bonardi RA, Oliveira JR. Doença de Crohn anoretoperineal.In:Habr-Gama A, Barone B, editores. Atualização em Coloproctologia. São Paulo: 1995. p. 189-196.

39. Costa ACT, Bettarello A. Editorial:Doença Inflamatória Crônica do Intestino e Risco de Câncer. Revista do Hospital das Clínicas de São Paulo, 1981;36:189-193.

40. Fiss E, Steinwaurz F, Vannucci AB, Succi CM. Derrame pleural: uma complicação extra-intestinal da doença de Crohn. J. Pneumologia. 2002; 28: 285-287.

41. Godoy AC, Habr-Gama A,Mauro E. Moléstia de Crohn primária do intetino grosso. Revista Paulista de Medicina 1965;66:273-280.

42. Habr-Gama A, Teixeira MG. Doença de Crohn do intestino delgado e do intestino grosso. In:Raia AA, Zerbini EJ. Clínica Cirúrgica - Alípio Correia Netto. 4a ed. São Paulo: Sarvier; 1988. p-419-432.

43. Habr-Gama A. Doença de Crohn. In:Moreira H. Atualização em Coloproctologia. $1^{a}$ ed. Goiânia: Escaleno Ltda; 1992. p145-156. 
Rev bras Coloproct Julho/Setembro, 2009
Interação da Gestação na Atividade da Doença Inflamatória Intestinal e sua Influência sobre o Prognóstico Gestacional e na Fecundidade Leonardo Corrêa de Oliveira Rodrigues e Cols.
Vol. 29
44. Jorge JMN, Habr-Gama A. Proctocolectomia total no tratamento das doenças inflamatórias intestinais. In:Habr-Gama A, Barone B. Atualização em Coloproctologia. São Paulo: 1995. p-111-120.

45. Kotze LMS Doença de Crohn - Experiência pessoal. Revista Brasileira de Coloproctologia 1991; 11: 9-14.

46. Laudanna AA. Importância das doenças inflamatórias intestinais na gastroenterologia atual. In: Habr-Gama A, Barone B. Atualização em Coloproctologia. São Paulo: 1995. p-1-7.

47. Silva AL, Melanazo HV, Queiroz E, Andrade DO. Doença de Crohn. Revista Brasileira de Medicina 1990; 47:319-334.

48. Magalhães AFN. Doença de Crohn. In:Gastroenterologia Clínica. $2^{a}$ ed. Rio de Janeiro: Ed. Guanabara Koogan S.A; 1988.p653-663. Moura-Filho GL, Ribeiro S. Manifestações articulares da doença inflamatória intestinal. Arq Gastroenterol. 1985; 22: 186-191.

49. Teixeira MG, Habr-Gama A. Tratamento cirúrgico da doença de Crohn intestinal. In: Habr-Gama A, Barone B. Atualização em Coloproctologia. São Paulo: 1995. p-173-187.

50. Teixeira MG. Tratamento cirúrgico da doença de Crohn. São Paulo, 2000; Tese de Livre Docência USP.

51. Olsen KO, Juul S, Berndtsson I et al. Ulcerative colitis: female fecundity before diagnosis, during disease, and after surgery compared with a population sample. Gastroenterology 2002; 122: 15-19.

52. Moody G, Probert C, Srivasta, et al. Sexual dysfunction among patients with inflammatory bowel disease. Digestion 1993; 52: 256-260.

53. Hudson M, Flett G, Sinclair TS, et al. Fertility and pregnancy in inflammatory bowel disease. Int J Gynaecol Obstet 1997; 58: 229-237.

54. Marri SR, Ahn C, Buchman AL. Voluntary childlessness is increased in women with inflammatory bowel disease. Inflamm Bowel Dis. 2007 May; 13(5); 591-599.

55. Fielding JF, Cooke WT. Pregnancy and Crohn's disease. BMJ 1970; 2: 76-77.

56. Fielding JF. Pregnancy and inflammatory bowel disease. Ir J Med Sci 1982; 151:194-202.

57. Alstead EM. Inflammatory bowel disease and pregnancy. Postgrad Med J 2002; 78: 23-26.

58. Nielsen $\mathrm{OH}$, Andreasson B, Bondensen S, Jarnum S. Pregnancy in ulcerative colitis. Scand J Gastroenterology 1983; 18: 735742.

59. Homan WP, Thorbjarnarson B. Crohn disease and pregnancy. Arch Surg 1976; 111: 545-547.

60. Nwokolo CU, Tan WC, Andrews HA, et al. Surgical resection in parous patients with distal ileal and colonic Crohn's disease. Gut 1994; 35: 220-223.
61. Katz JA. Pregnancy and inflammatory bowel disease. Curr Opin Gastroenterol 2004; 20; 328-332.

62. Norgard B, Fonager K, Sorensen HT, Olsen J. Birth outcomes of women with ulcerative colitis: a nationwide Danish cohort study. Am J Gastroenterol 2000; 95: 3165-3170.

63. Kornfeld D, Cnattingius S, Ekbom A. Pregnancy outcomes in women with inflammatory bowel disease - A populationbased cohort study. Am J Obstet Gynecol 1997; 177: 942946.

64. Riis L, Vind I, Politi P et al. On behalf of the European Collaborative study group on inflammatory bowel disease (EC-IBD). Does pregnancy change the disease course? A study in a European Cohort of patients with inflammatory bowel disease. Am J Gastroenterol 2006; 101: 1539-1545.

65. Ira M, Hanan, MD. Inflammatory bowel disease in the pregnant woman. Comprehensive Therapy 1993; 19(3): 91-95.

66. Fedorkow DM, Persaud D, Nimrod MB. Inflammatory bowel disease: a controlled study of late pregnancy outcome. Am J Obstet Gynecol 1989; 160: 998-1001.

67. Moser MA, Okun NB, Mayes DC, Bayley RJ. Crohn's disease pregnancy and birth weight. Am J Gastroenterol 2000; 95: 1021-1026.

68. Diav-Citrin O, Park YH, Veerasuntharam G, et al. The safety of mesalamine in human pregnancy: a prospective controlled cohort study. Gastroenterology 1998; 114: 23-28.

69. Colombel JF, Brabant G, Gubler MC, et al. Renal insufficiency in infant: side- effect of prenatal exposure to mesalazine? Lancet 1994; 344: 620- 621.

70. Rodriguez- Pinilla E, Martinez-Frias ML. Corticosteroids during pregnancy and oral clefts: a case- control study. Teratology 1998; 58: 2-5.

71. Carmichael SL, Shaw GM. Maternal corticosteroid use and risk of selected congenital anomalities. Am J Med Genet 1999; 86: 242-244.

72. Park- Wyllie L, Mazzotta P, Pastuszak A, et al. Birth defects after maternal exposure to corticosteroids: prospective cohort study and meta- analysis of epidemiological studies. Teratology 2000; 62: 385-392.

\section{Endereço para correspondência:}

\section{LEONARDO CORREAA DE OLIVEIRA RODRIGUES}

Rua Santa Clara, 335 / apto. 701 - Copacabana

CEP: 22041-010 - Rio de Janeiro - RJ

E-mail: drleorodrigues@ig.com.br 